\title{
Asymptotic Behavior of some Rational Difference Equations
}

\author{
E.M. Elabbasy \\ Department of Mathematics, \\ Faculty of Science, Mansoura Uni- \\ versity, Mansoura, 35516 \\ Egypt
}

\author{
A.A. El-Biaty \\ Department of Mathematics, The \\ Faculty of Education, University of Tikrit \\ Iraq
}

\begin{abstract}
In this difference equation, Stability, Periodicity, boundedness, global Stability.
\end{abstract}

We investigate some qualitative behavior of the solutions of the difference equation

$x_{n+1}=a x_{n-\ell}+\sum_{i=0}^{k} \alpha_{i} x_{n-i} / \sum_{i=0}^{k} \beta_{i} x_{n-i}$ where the the

initial conditions $x_{-r}, x_{-r+1}, \ldots, x_{0}$ are arbitrary positive real numbers such that $r=\max \{\ell, k\}$ where $i, r \in\{0,1, \ldots\}$ and $a, \alpha_{i}, \beta_{i}$ are positive constants.

\section{Keywords}

difference equation, Stability, Periodicity, boundedness.

\section{INTRODUCTION}

In this paper we deal with some properties of the solutions of the difference equation

$$
x_{n+1}=a x_{n-\ell}+\frac{\sum_{i=0}^{k} \alpha_{i} x_{n-i}}{\sum_{i=0}^{k} \beta_{i} x_{n-i}}, \quad n=0,1,2, \ldots,(1.1)
$$

where the the initial conditions $x_{-r}, x_{-r+1}, \ldots, x_{0}$ are arbitrary positive real numbers such that $r=\max \{\ell, k\}$ where $i, r \in\{0,1, \ldots\}$ and $a, \alpha_{i}, \beta_{i}$ are positive constants. There is a class of nonlinear difference equations, known as the rational difference equations, each of which consists of the ratio of two polynomials in the sequence terms in the same from. there has been a lot of work concenring the global asympototic of solutions of rational difference equations [2], [3], [4], [7], [8], [11] and [12].

Many reseaches have investigated the behavior of the solution of difference equation for example:

Kulenovic1 et al.[13] has studied the global asymptotic stability of solutions of the equation

$$
x_{n+1}=\frac{\alpha x_{n}+\beta x_{n-1}}{\gamma x_{n}+\delta x_{n-1}} .
$$

M. Saleh et al.[15] investigated the periodic character and the global stability of all positive solutions of the equation

$$
x_{n+1}=\frac{\beta x_{n}+\gamma x_{n-k}}{B x_{n}+C x_{n-k}} .
$$

Our aim in this paper is to extend and generalize the work in [1], [6], [10], [13], [15], [16] and [17]. That is, we will investigate the global behavior of (1.1) including the asymptotical stability of equilibrium points, the existence of bounded solution, the existence of period two solution and investigate the oscillation property of the recursive sequence of Eq. (1.1).

Now we recall some well-known results, which will be useful in the investigation of (1.1) and which are given in [9].

Let I be an interval of real numbers and let

$F: I^{k+1} \rightarrow I$,

where $F$ is a continuous function. Consider the difference equation

$$
x_{n+1}=F\left(x_{n}, x_{n-1}, \ldots, x_{n-k}\right), n=0,1,2, \ldots, \text { (1.2) }
$$

with the initial condition $x_{-k}, x_{-k+1}, \ldots, x_{0} \in I$.

Definition 1 (Equilibrium Point)

A point $\bar{x} \in I$ is called an equilibrium point of Eq. (1.2) if $\bar{x}=f(\bar{x}, \bar{x}, \ldots, \bar{x})$.

That is, $x_{n}=\bar{x}$ for $n \geq 0$, is a solution of Eq. (1.2), or equivalently, $\bar{x}$ is a fixsed point of $f$.

Definition 2 (Stability)

Let $\bar{x} \in(0, \infty)$ be an equilibrium point of Eq. (1.2). Then

i) An equilibrium point $x$ of Eq. (1.2) is called locally stable if for every $\varepsilon>0$ there exists $\delta>0$ such that, if $x_{-r}, x_{-r+1}, \ldots, x_{0} \in(0, \infty)$ with $\left|x_{-r}-\bar{x}\right|+\left|x_{-r+1}-\bar{x}\right|+\ldots+\left|x_{0}-\bar{x}\right|<\delta, \quad$ then $\left|x_{n}-\bar{x}\right|<\varepsilon$ for all $n \geq-r$.

ii) An equilibrium point $\bar{x}$ of Eq. (1.2) is called locally asymptotically stable if $\bar{x}$ is locally stable and there exists 
$\gamma>0$ such that, if $x_{-r}, x_{-r+1}, \ldots, x_{0} \in(0, \infty)$ with $\left|x_{-r}-\bar{x}\right|+\left|x_{-r+1}-\bar{x}\right|+\ldots+\left|x_{0}-\bar{x}\right|<\gamma$, then

$\lim _{n-\infty} x_{n}=\bar{x}$

iii) An equilibrium point $\bar{x}$ of Eq. (1.2) is called a global attractor if for every $x_{-r}, x_{-r+1}, \ldots, x_{0} \in(0, \infty)$ we have $\lim _{n \rightarrow \infty} x_{n}=\bar{x}$

iv) An equilibrium point $\bar{x}$ of Eq. (1.2) is called globally asymptotically stable if $\bar{x}$ is locally stable and a global attractor.

v) An equilibrium point $\bar{x}$ of Eq. (1.2) is called unstable if $x$ is not locally stable.

\section{Definition 3 (Permanence)}

Eq. (1.2) is called permanent if there exists numbers $m$ and $M$ with $0<m<M<\infty$ such that for any initial conditions $x_{-r}, x_{-r+1}, \ldots, x_{0} \in(0, \infty)$ there exists a positive integer $N$ which depends on the initial conditions such that

$m \leq x_{n} \leq M$ forall $n \geq-N$.

\section{Definition 4 (Periodicity)}

A sequence $\left\{x_{n}\right\}_{n=-r}^{\infty}$ is said to be periodic with period $p$ if $\quad x_{n+p}=x_{n} \quad$ for all $\quad n \geq-r . \quad$ A sequence $\left\{x_{n}\right\}_{n=-r}^{\infty}$ is said to be periodic with prime period $p$ if $p$ is the smallest positive integer having this property.

The linearized equation of Eq. (1.2) about the equilibrium point $\bar{x}$ is defined by the equation

$$
z_{n+1}=\sum_{i=0}^{k} p_{i} z_{n-i}
$$

where

$p_{i}=\frac{\partial F(\bar{x}, \bar{x}, \ldots, \bar{x},)}{\partial x_{n-i}}, \quad i=0,1, \ldots, k$.

The characteristic equation associated with Eq. (1.3) is

$\lambda^{k+1}-p_{0} \lambda^{k}-p_{1} \lambda^{k-1}-\ldots-p_{k-1} \lambda-p_{k}=0$.

Theorem 1.2 [9]. Assume that $F$ is a $C^{1}-$ function and let $x$ be an equilibrium point of Eq. (1.2). Then the following statements are true:

i) If all roots of Eq. (1.4) lie in the open unit disk $|\lambda|<1$, then he equilibrium point $\bar{x}$ is locally asymptotically stable.

ii) If at least one root of Eq. (1.4) has absolute value greater than one, then the equilibrium point $\bar{x}$ is unstable.

iii) If all roots of Eq. (1.4) have absolute value greater than one, then the equilibrium point $\bar{x}$ is a source.

Theorem 1.3 [14] Assume that $p_{i} \in R, i=1,2, \ldots, k$. Then $\sum_{i=1}^{k}\left|p_{i}\right|<1$

is a sufficient condition for the asymptoticcally stable of Eq (1.5)

$y_{n+k}+p_{1} y_{n+k-1}+\ldots+p_{k} y_{n}=0, n=0,1, \ldots(1.5)$.

\section{LOCAL STABILITY OF THE EQUILIBRIUM POINT OF EQ. (1.1)}

In this section we investigate the local stability character of the solutions of Eq. (1.1). Eq. (1.1) has a unique nonzero equilibrium point

$\bar{x}=a \bar{x}+\frac{\sum_{i=0}^{k} \alpha_{i} \bar{x}}{\sum_{i=0}^{k} \beta_{i} \bar{x}}$,

if $1>a$, of the Eq. (1) has only positive equilibrium point $\bar{x}$ is given by

$$
\bar{x}=\frac{\sum_{i=0}^{k} \alpha_{i}}{\sum_{i=0}^{k} \beta_{i}(1-a)} \text {. }
$$

Let

$$
\begin{aligned}
G & =\sum_{i=0}^{k} \alpha_{i}, Q=\sum_{i=0}^{k} \beta_{i}, \\
G^{i} & =\sum_{\substack{j=0 \\
j \neq i}}^{k} \alpha_{j} \text { and } Q^{i}=\sum_{\substack{j=0 \\
j \neq i}}^{k} \beta_{j} .
\end{aligned}
$$

Then, we get

$$
\bar{x}=\frac{G}{Q(1-a)} \text {. }
$$

Let $f:(0, \infty)^{k+1} \rightarrow(0, \infty)$ be a function defined by

$$
f\left(u_{0}, u_{1}, \ldots, u_{k}, v\right)=a v+\frac{\sum_{i=0}^{k} \alpha_{i} u_{i}}{\sum_{i=0}^{k} \beta_{i} u_{i}} .
$$

Therefore it follows that 


$$
\begin{aligned}
& \frac{\partial f\left(u_{0}, u_{1}, \ldots, u_{k}, v\right)}{\partial v}=a, \\
& \frac{\partial f\left(u_{0}, u_{1}, \ldots, u_{k}, v\right)}{\partial u_{0}}=\frac{\alpha_{0}\left(\sum_{i=1}^{k} \beta_{i} u_{i}\right)-\beta_{0}\left(\sum_{i=1}^{k} \alpha_{i} u_{i}\right)}{\left[\sum_{i=0}^{k} \beta_{i} u_{i}\right]^{2}}, \\
& \frac{\partial f\left(u_{0}, u_{1}, \ldots, u_{k}, v\right)}{\partial u_{1}}=\frac{\alpha_{1}\left(\sum_{i=0, i \neq 1}^{k} \beta_{i} u_{i}\right)-\beta_{1}\left(\sum_{i=0, i \neq 1}^{k} \alpha_{i} u_{i}\right)}{\left[\sum_{i=0}^{k} \beta_{i} u_{i}\right]^{2}} \\
& \frac{\partial f\left(u_{0}, u_{1}, \ldots, u_{k}, v\right)}{\partial u_{j}}=\frac{\cdot}{\alpha_{j}\left(\sum_{i=0, i \neq j}^{k} \beta_{i} u_{i}\right)-\beta_{j}\left(\sum_{i=0, i \neq j}^{k} \alpha_{i} u_{i}\right)}
\end{aligned}
$$

and

$$
\frac{\partial f\left(u_{0}, u_{1}, \ldots, u_{k}, v\right)}{\partial u_{k}}=\frac{\alpha_{k}\left(\sum_{i=0}^{k-1} \beta_{i} u_{i}\right)-\beta_{k}\left(\sum_{i=0}^{k-1} \alpha_{i} u_{i}\right)}{\left[\sum_{i=0}^{k} \beta_{i} u_{i}\right]^{2}} .
$$

Then we see that

$$
\begin{aligned}
& \frac{\partial f(\bar{x}, \bar{x}, \ldots, \bar{x}, \bar{x})}{\partial v}=a \\
& \frac{\partial f(\bar{x}, \bar{x}, \ldots, \bar{x}, \bar{x})}{\partial u_{0}}=\frac{\left(\alpha_{0} \sum_{i=1}^{k} \beta_{i}-\beta_{0} \sum_{i=1}^{k} \alpha_{i}\right)(1-a)}{Q G}, \\
& \frac{\partial f(\bar{x}, \bar{x}, \ldots, \bar{x}, \bar{x})}{\partial u_{1}}=\frac{\left(\alpha_{1} \sum_{i=0, i \neq 1}^{k} \beta_{i}-\beta_{1} \sum_{i=0, i \neq 1}^{k} \alpha_{i}\right)(1-a)}{Q G},
\end{aligned}
$$

$$
\frac{\partial f(\bar{x}, \bar{x}, \ldots, \bar{x}, \bar{x})}{\partial u_{j}}=\frac{\left(\alpha_{j} \sum_{i=0, i \neq j}^{k} \beta_{i}-\beta_{j} \sum_{i=0, i \neq j}^{k} \alpha_{i}\right)(1-a)}{Q G},
$$

and

$$
\frac{\partial f(\bar{x}, \bar{x}, \ldots, \bar{x}, \bar{x})}{\partial u_{k}}=\frac{\left(\alpha_{k} \sum_{i=0}^{k-1} \beta_{i}-\beta_{k} \sum_{i=0}^{k-1} \alpha_{i}\right)(1-a)}{Q G} .
$$

Then the linearized equation of (1.1) about $\bar{x}$ is

$$
z_{n+1}=\sum_{i=0}^{k} p_{i} z_{n-i} \text {. }
$$

Theorem 2.1 Assume that $\sum_{i=0}^{k}\left(\alpha_{i} Q^{i}-\beta_{i} G^{i}\right)<G Q$.

Than the equilibrium point of Eq. (1.1) is locally stable.

Proof. It is follows by Theorem(1.3) that, Eq. (2.2) is locally stable if

$$
\left|p_{k}\right|+\ldots+\left|p_{j}\right|+\ldots+\left|p_{1}\right|+\left|p_{0}\right|<1 \text {. }
$$

That is

$$
\begin{aligned}
& |a|+\sum_{i=0}^{k}\left|\frac{\left(\alpha_{i} Q^{i}-\beta_{i} G^{i}\right)(1-a)}{Q G}\right|<1 . \\
& \text { If } \\
& \alpha_{i} Q^{i}>\beta_{i} G^{i},
\end{aligned}
$$
this implies that

$$
a Q G+\sum_{i=0}^{k}\left(\alpha_{i} Q^{i}-\beta_{i} G^{i}\right)(1-a)<Q G .
$$

Thus

$$
\sum_{i=0}^{k}\left(\alpha_{i} Q^{i}-\beta_{i} G^{i}\right)<G Q .
$$

Hence, the proof is completed.

Example 2.1 Consider the difference equation $x_{n+1}=0.5 x_{n-1}+\frac{0.125 x_{n}+0.25 x_{n-1}}{0.5 x_{n}+0.5 x_{n-1}}$, where

$k=1, \ell=1, a=0.5, \alpha_{0}=0.125, \alpha_{1}=0.25$, $\beta_{0}=0.5, \beta_{1}=0.5$.

Figure(2.1), shows that the equilibrium point of Eq. (1.1) has 
locally stable, with initial data $x_{n-1}=0.1, x_{0}=6.1$.

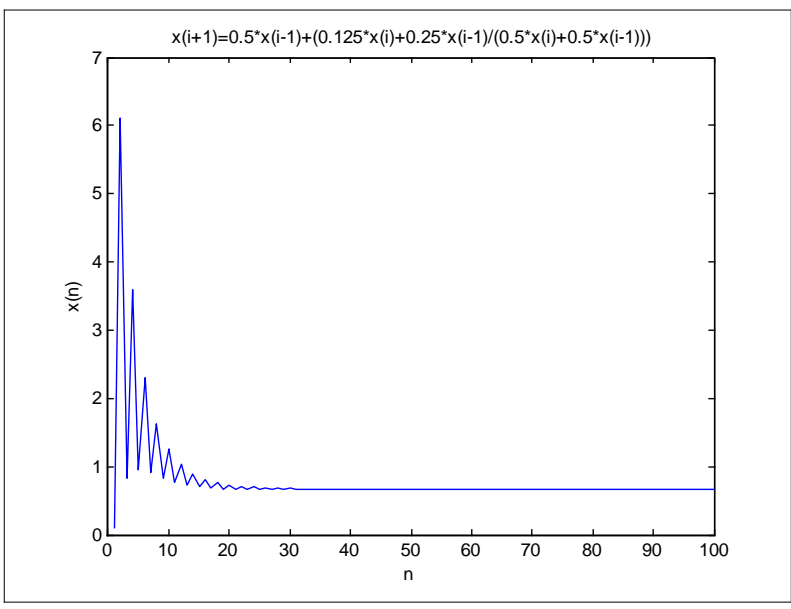

Fig 2.1

\section{PERIODIC SOLUTIONS OF EQ. (1.1)}

In this section we investigate the periodic character of the positive solutions of Eq. (1.1).

Theorem 3.1 Eq. (1.1) has positive prime priod two solution If
(i) $\ell-o d d, k-o d d$ and
$(\delta-\gamma)(\mu-\lambda)(1-a)>4 \gamma$,

(ii) $\ell$-odd, $k-$ even and

$(\delta-\gamma)(\mu-\lambda)(1-a)>4 \gamma$,

(iii) $\ell$-even, $k$-odd and

$(\delta-\gamma)(\mu-\lambda)(1+a)>4(\delta a \mu+\lambda \gamma)$,

(iv) $\ell$-even, $k$-even and

$(\delta-\gamma)(\mu-\lambda)(1+a)>4(\delta a \mu+\lambda \gamma)$.

Proof. For case(i) assume that there exists a prime periodtwo solution $\ldots, p, q, p, q, \ldots$

of (1.1). Let $\quad x_{n}=q, x_{n+1}=p . \quad$ Since

$\ell$-odd, $k$-odd we have $\quad x_{n-\ell}=p, x_{n-k}=p$.

Thus, from Eq. (1.1), we get

$$
\begin{array}{r}
p=a p+\frac{\alpha_{0} q+\alpha_{1} p+\ldots+\alpha_{k} p}{\beta_{0} q+\beta_{1} p+\ldots+\beta_{k} p}, \\
q=a q+\frac{\alpha_{0} p+\alpha_{1} q+\ldots+\alpha_{k} q}{\beta_{0} p+\beta_{1} q+\ldots+\beta_{k} q} .
\end{array}
$$

Let

$\alpha_{0}+\alpha_{2}+\ldots+\alpha_{k-1}=\gamma$,

and $\alpha_{1}+\alpha_{3}+\ldots+\alpha_{k}=\delta$,

and let

$\beta_{0}+\beta_{2}+\ldots+\beta_{k-1}=\mu$,

and

$\beta_{1}+\beta_{3}+\ldots+\beta_{k}=\lambda$.

Than

$p=a p+\frac{\gamma q+\delta p}{\mu q+\lambda p}$

and

$q=a q+\frac{\not p+\delta q}{\mu p+\lambda q}$

Than

$\mu p q+\lambda p^{2}=a \mu p q+a \lambda p^{2}+\gamma q+\delta p$,

and

$\mu p q+\lambda q^{2}=a \mu p q+a \lambda q^{2}+\not p+\delta q$.

Subtracting (3.3) from (3.4) gives

$$
\lambda(1-a)\left(p^{2}-q^{2}\right)=(\delta-\gamma)(p-q) \text {. }
$$

Since $p \neq q$, we have

$p+q=\frac{\delta-\gamma}{\lambda(1-a)}$.

Also, since $p$ and $q$ are positive, $(\delta-\gamma), \lambda(1-a)$ shuold be positive. Again, adding (3.3) and (3.4) yields

$$
\begin{aligned}
& 2 \mu p q+\lambda\left(p^{2}+q^{2}\right)=2 a \mu p q+ \\
& a \lambda\left(p^{2}+q^{2}\right)+\gamma(p+q)+\delta(p+q) .
\end{aligned}
$$

It follows by (3.5), (3.6) and the relation

$$
p^{2}+q^{2}=(p+q)^{2}-2 p q, \quad \forall p, q \in \mathrm{R},
$$

that

$$
p q=\frac{\gamma(\delta-\gamma)}{\lambda^{2}(1-a)^{3}(\mu-\lambda)} .
$$

It is clear now, from Eq. (3.5) and Eq. (3.7) that $p$ and $q$ are the two distinct roots of the quadratic equation

$t^{2}-\left(\frac{\delta-\gamma}{\lambda(1-a)}\right) t+\frac{\gamma(\delta-\gamma)}{\lambda^{2}(1-a)^{3}(\mu-\lambda)}=0$,

and so

$\left(\frac{\delta-\gamma}{\lambda(1-a)}\right)^{2}-\frac{4 \gamma(\delta-\gamma)}{\lambda^{2}(1-a)^{3}(\mu-\lambda)}>0$,

which is equivalent to

$$
(\delta-\gamma)(\mu-\lambda)(1-a)>4 \gamma \text {. }
$$

The proof follows by induction. The cases where (ii), holds is similar and will be omitted.

For case (iii) assume that there exists a prime period-two solution

$\ldots, p, q, p, q, \ldots$

of (1.1). Let $\quad x_{n}=q, x_{n+1}=p . \quad$ Since

$\ell$-even, $k$-odd $\quad$ we have $\quad x_{n-\ell}=q, x_{n-k}=p$.

Thus, from Eq. (1.1), we get 
$p=a q+\frac{\alpha_{0} q+\alpha_{1} p+\ldots+\alpha_{k} p}{\beta_{0} q+\beta_{1} p+\ldots+\beta_{k} p}$,

$q=a p+\frac{\alpha_{0} p+\alpha_{1} q+\ldots+\alpha_{k} q}{\beta_{0} p+\beta_{1} q+\ldots+\beta_{k} q}$.

Let

$\alpha_{0}+\alpha_{2}+\ldots+\alpha_{k-1}=\gamma$,

where

and

$\alpha_{1}+\alpha_{3}+\ldots+\alpha_{k}=\delta$,

and let

$\beta_{0}+\beta_{2}+\ldots+\beta_{k-1}=\mu$,

and

$\beta_{1}+\beta_{3}+\ldots+\beta_{k}=\lambda$.

Than

$p=a q+\frac{\gamma q+\delta p}{\mu q+\lambda p}$

and

$q=a p+\frac{\not p+\delta q}{\mu p+\lambda q}$

Than

$\mu p q+\lambda p^{2}=a \mu q^{2}+a \lambda p q+\gamma q+\delta p$,

and

$\mu p q+\lambda q^{2}=a \mu p^{2}+a \lambda p q+\not p+\delta q$.

Subtracting (3.8) from (3.9) gives

$(\lambda+a \mu)\left(p^{2}-q^{2}\right)=(\delta-\gamma)(p-q)$.

Since $p \neq q$, we have

$p+q=\frac{\delta-\gamma}{\lambda+a \mu}$

Also, since $p$ and $q$ are positive, $(\delta-\gamma)$ shuold be positive. Again, adding (3.8) and (3.9) yields

$2 \mu p q+\lambda\left(p^{2}+q^{2}\right)=a \mu\left(p^{2}+q^{2}\right)+$

$2 a p p q+\gamma(p+q)+\delta(p+q)$.

It follows by (3.10), (3.11) and the relation

$p^{2}+q^{2}=(p+q)^{2}-2 p q, \quad \forall p, q \in \mathrm{R}$,

that

$p q=\frac{(\delta-\gamma)(\delta a \mu+\lambda \gamma)}{(1+a)(\mu-\lambda)(\lambda-a \mu)^{2}}$.

It is clear now, from Eq. (3.10) and Eq. (3.12) that $p$ and $q$ are the two distinct roots of the quadratic equation

$t^{2}-\left(\frac{\delta-\gamma}{\lambda+a \mu}\right) t+\frac{(\delta-\gamma)(\delta a \mu+\lambda \gamma)}{(1+a)(\mu-\lambda)(\lambda-a \mu)^{2}}=0$

and so

$\left(\frac{\delta-\gamma}{\lambda+a \mu}\right)^{2}-\frac{4(\delta-\gamma)(\delta a \mu+\lambda \gamma)}{(1+a)(\mu-\lambda)(\lambda-a \mu)^{2}}>0$,

which is equivalent to

$(\delta-\gamma)(\mu-\lambda)(1+a)>4(\delta a \mu+\lambda \gamma)$.
The proof follows by induction. The cases where (iv), holds is similar and will be omitted. Now the proof is completed.

Example 3.1 Consider the difference equation

$$
\begin{aligned}
& x_{n+1}=0.5 x_{n-1}+\frac{0.002 x_{n}+0.5 x_{n-1}}{0.9 x_{n-2}+0.2 x_{n-1}}, \\
& k-o d d=1, \ell-\text { odd }, a=0.5, \alpha_{0}=0.002,
\end{aligned}
$$$$
\alpha_{1}=0.5, \beta_{0}=0.9, \beta_{1}=0.2 \text {. }
$$

Figure(3.1), shows that Eq. (1.1) which is periodic with period two $x_{-1}=0.7, x_{0}=5.3$. Where the initial data satisfies condition(3.1) of Theorem(3.1) (seeTable

\begin{tabular}{|c|c|c|c|c|c|c|c|c|c|}
\hline$n$ & $x(n)$ & $n$ & $x(n)$ & $n$ & $x(n)$ & $n$ & $x(n)$ & $n$ & $x(n)$ \\
\hline 1 & 0.7000 & 17 & 0.0392 & 33 & 0.0255 & 49 & 0.0253 & 65 & 0.0253 \\
\hline 2 & 5.3000 & 18 & 4.7454 & 34 & 4.8832 & 50 & 4.8862 & 66 & 4.8863 \\
\hline 3 & 0.4319 & 19 & 0.0337 & 35 & 0.0254 & 51 & 0.0253 & 67 & 0.0253 \\
\hline 4 & 4.4801 & 20 & 4.7954 & 36 & 4.8844 & 52 & 4.8863 & 68 & 4.8863 \\
\hline 5 & 0.2773 & 21 & 0.0303 & 37 & 0.0254 & 53 & 0.0253 & 69 & 0.0253 \\
\hline 6 & 4.1959 & 22 & 4.8286 & 38 & 4.8852 & 54 & 4.8863 & 70 & 4.8863 \\
\hline 7 & 0.1832 & 23 & 0.0283 & 39 & 0.0253 & 55 & 0.0253 & 71 & 0.0253 \\
\hline 8 & 4.1877 & 24 & 4.8501 & 40 & 4.8856 & 56 & 4.8863 & 72 & 4.8863 \\
\hline 9 & 0.1241 & 25 & 0.0271 & 41 & 0.0253 & 57 & 0.0253 & 73 & 0.0253 \\
\hline 10 & 4.3000 & 26 & 4.8638 & 42 & 4.8859 & 58 & 4.8863 & 74 & 4.8863 \\
\hline 11 & 0.0866 & 27 & 0.0264 & 43 & 0.0253 & 59 & 0.0253 & 75 & 0.0253 \\
\hline 12 & 4.4425 & 28 & 4.8724 & 44 & 4.8861 & 60 & 4.8863 & 76 & 4.8863 \\
\hline 13 & 0.0629 & 29 & 0.0259 & 45 & 0.0253 & 61 & 0.0253 & 77 & 0.0253 \\
\hline 14 & 4.5715 & 30 & 4.8778 & 46 & 4.8862 & 62 & 4.8863 & 78 & 4.8863 \\
\hline 15 & 0.0482 & 31 & 0.0257 & 47 & 0.0253 & 63 & 0.0253 & 79 & 0.0253 \\
\hline 16 & 4.6725 & 32 & 4.8811 & 48 & 4.8862 & 64 & 4.8863 & 80 & 4.8863 \\
\hline
\end{tabular}
3.1)

Table 3.1

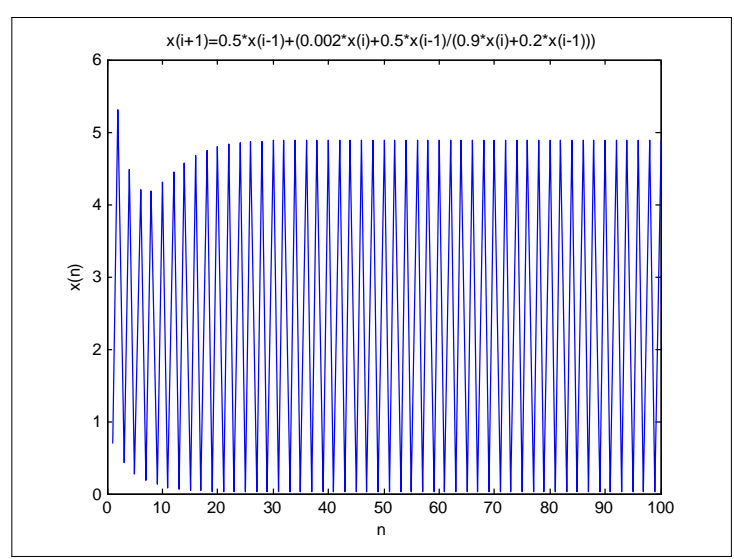

Fig3.2

Example 3.2 Consider the difference equation

$$
\begin{aligned}
& x_{n+1}=0.005 x_{n-2}+\frac{0.1 x_{n}+5 x_{n-1}}{9 x_{n-2}+0.02 x_{n-1}}, \\
& k-\text { odd }=1, \ell-\text { even, } a=0.005, \alpha_{0}=0.1, \\
\text { where } \quad & \alpha_{1}=5, \beta_{0}=9, \beta_{1}=0.02 .
\end{aligned}
$$

Figure(3.2), shows that Eq. (1.1) which is periodic with period two. Where the initial data satisfies condition(3.2) of Theorem(3.1)

$$
x_{n-2}=6.7, x_{-1}=3.9, x_{0}=2.9 \text {. }
$$


(seeTable 3.2)

\begin{tabular}{|c|c|c|c|c|c|c|c|c|c|}
\hline$n$ & $x(n)$ & $n$ & $x(n)$ & $n$ & $x(n)$ & $n$ & $x(n)$ & $n$ & $x(n)$ \\
\hline 1 & 6.7000 & 17 & 4.6513 & 33 & 4.6290 & 49 & 4.6290 & 65 & 4.6290 \\
\hline 2 & 3.9000 & 18 & 0.5565 & 34 & 0.5523 & 50 & 0.5523 & 66 & 0.5523 \\
\hline 3 & 2.9000 & 19 & 4.6174 & 35 & 4.6290 & 51 & 4.6290 & 67 & 4.6290 \\
\hline 4 & 1.0684 & 20 & 0.5519 & 36 & 0.5523 & 52 & 0.5523 & 68 & 0.5523 \\
\hline 5 & 1.6253 & 21 & 4.6209 & 37 & 4.6290 & 53 & 4.6290 & 69 & 4.6290 \\
\hline 6 & 0.5417 & 22 & 0.5515 & 38 & 0.5523 & 54 & 0.5523 & 70 & 0.5523 \\
\hline 7 & 1.7153 & 23 & 4.6276 & 39 & 4.6290 & 55 & 4.6290 & 71 & 4.6290 \\
\hline 8 & 0.3550 & 24 & 0.5521 & 40 & 0.5523 & 56 & 0.5523 & 72 & 0.5523 \\
\hline 9 & 2.6942 & 25 & 4.6297 & 41 & 4.6290 & 57 & 4.6290 & 73 & 4.6290 \\
\hline 10 & 0.3512 & 26 & 0.5523 & 42 & 0.5523 & 58 & 0.5523 & 74 & 0.5523 \\
\hline 11 & 4.2277 & 27 & 4.6295 & 43 & 4.6290 & 59 & 4.6290 & 75 & 4.6290 \\
\hline 12 & 0.4824 & 28 & 0.5524 & 44 & 0.5523 & 60 & 0.5523 & 76 & 0.5523 \\
\hline 13 & 4.8260 & 29 & 4.6291 & 45 & 4.6290 & 61 & 4.6290 & 77 & 4.6290 \\
\hline 14 & 0.5593 & 30 & 0.5523 & 46 & 0.5523 & 62 & 0.5523 & 78 & 0.5523 \\
\hline 15 & 4.7622 & 31 & 4.6290 & 47 & 4.6290 & 63 & 4.6290 & 79 & 4.6290 \\
\hline 16 & 0.5656 & 32 & 0.5523 & 48 & 0.5523 & 64 & 0.5523 & 80 & 0.5523 \\
\hline
\end{tabular}

Fig3.2

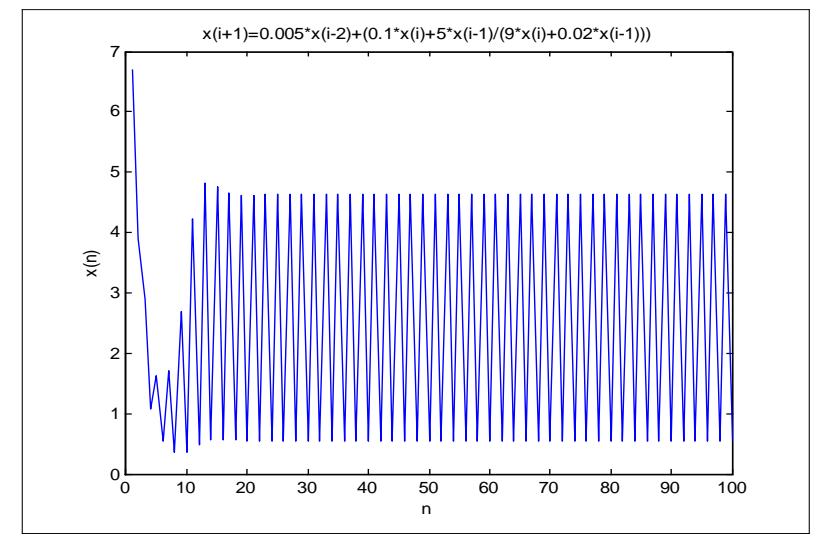

Fig3.3

\section{BOUNDED SOLUTIONS OF EQ. (1.1)}

Our aim in this section we investigate the boundedness of the positive solutions of Eq. (1.1).

Theorem 4.1 For Eq. (1.1) every solution is bounded if $1>a$.

Proof. Let $\left\{x_{n}\right\}_{n=-k}^{\infty}$ be a solution of Eq. (1.1). It follows from Eq. (1.1) that

$$
\begin{aligned}
x_{n+1} & =a x_{n-\ell}+\frac{\sum_{i=0}^{k} \alpha_{i} x_{n-i}}{\sum_{i=0}^{k} \beta_{i} x_{n-i}} \\
& =a x_{n-\ell}+\frac{\alpha_{0} x_{n}}{\sum_{i=0}^{k} \beta_{i} x_{n-i}}+\frac{\alpha_{1} x_{n-1}}{\sum_{i=0}^{k} \beta_{i} x_{n-i}}+\ldots+\frac{\alpha_{k} x_{n-k}}{\sum_{i=0}^{k} \beta_{i} x_{n-i}} \\
& \leq a x_{n-\ell}+\frac{\alpha_{0} x_{n}}{\beta_{0} x_{n}}+\frac{\alpha_{1} x_{n-1}}{\beta_{1} x_{n-1}}+\ldots+\frac{\alpha_{k} x_{n-k}}{\beta_{k} x_{-k}} \\
& =a x_{n-\ell}+\sum \frac{\alpha_{i}}{\beta_{i}} \text { for all } n \geq 1 .
\end{aligned}
$$

By using a comparison, we can write the right hand side as follows

$$
y_{n+1}=a y_{n-\ell}+\sum_{i=0}^{k} \frac{\alpha_{i}}{\beta_{i}},
$$

then

$$
y_{n}=a^{n} y_{-\ell}+\sum_{i=0}^{k} \frac{\alpha_{i}}{\beta_{i}},
$$

and this equation is locally stable because $1>a$, and converges to the equilibrium point

$$
\bar{y}=\frac{1}{(1-a)} \sum_{i=0}^{k} \frac{\alpha_{i}}{\beta_{i}} .
$$

Therefore

$$
\limsup _{n \rightarrow \infty} x_{n} \leq \frac{1}{(1-a)} \sum_{i=0}^{k} \frac{\alpha_{i}}{\beta_{i}} \text {. }
$$

Thus, for Eq. (1.1) every solutoin is bounded and the proof is completed.

Theorem 4.2 For Eq. (1b) every solution is unbounded if $1<a$.

Proof. Let $\left\{x_{n}\right\}_{n=-k}^{\infty}$ be a solution of Eq. (1.1). It follows from Eq. (1.1) then

$$
x_{n+1}=a x_{n-\ell}+\frac{\sum_{i=0}^{k} \alpha_{i} x_{n-i}}{\sum_{i=0}^{k} \beta_{i} x_{n-i}}>a x_{n-\ell} \text { for all } n \geq 1 \text {. }
$$

We can written as follows

$$
y_{n+1}=a y_{n-\ell},
$$

then

$$
y_{n}=a^{n} y_{-\ell},
$$

and the Eq. (4.1) is unstable because $1<a$, and

$\lim _{n \rightarrow \infty} y_{n}=\infty$.

Thus, the proof is completed.

\section{REFERENCES}

[1] R. DeVault, S. W. Schultz, On the dynamics of $x_{n+1}=\frac{\beta x_{n}+\gamma x_{n-1}}{B x_{n}+D x_{n-2}}, \quad$ Domm. Appl. Nonlinear Analysis, 12 (2005), 35-40.

[2] E. M. Elabbasy, H. El-Metwally and E. M. Elsayed, On the periodic nature of some max-type difference equations, Int. J. Math. Sci., 14 (2005), 2227-2239.

[3] E. M. Elabbasy, H. El-Metwally and E. M. Elsayed. On the difference equation $x_{n+1}=a x_{n}-\frac{b x_{n}}{c x_{n}-d x_{n-1}}$. 
Adv. Difference Equ., pages Art. ID, 10 (2006), 82579.

[4] E.M. Elabbasy, H. El-Metwally and E.M. Elsayed. Qualitative behavior of higher order difference equation. Soochow J. Math., 33(4) (2007), 861--873.

[5] E. M. Elabbasy, H. El-Metwally and E. M. Elsayed, On the difference equations $x_{n+1}=\frac{\alpha x_{n-k}}{\beta+\gamma \prod_{i=0}^{k} x_{n-i}}, \quad$ J. Conc. Appl. Math. 5(2) (2007), 101-113.

[6] E. M. Elabbasy, H. El-Metwally and E. M. Elsayed. On the differencee quation $x_{n+1}=\frac{\left(a_{0} x_{n}+a_{1} x_{n-1}+\ldots+a_{k} x_{n-k}\right)}{\left(b_{0} x_{n}+b_{1} x_{n-1}+\ldots+b_{k} x_{n-k}\right)}$, Mathematica Bohemica, 133 (2008), No.2, 133-147.

[7] S. N. Elaydi, An Introduction to difference equations, Undergraduate Texts in Mathematics, Springer, New York, NY, USA, (1996).

[8] M. A. El-Moneam, E. M. E. Zayed, Dynamics of the rational difference equation $x_{n+1}=A x_{n}+B x_{n-k}+C x_{n-\ell}+\frac{b x_{n} x_{n-k} x_{n-\ell}}{d x_{n-k}-e x_{n-\ell}}$, DCDIS Ser. A: Math. Anal. 21 (2014), 317--331.

[9] E.A. Grove, G. Ladas, Periodicities in nonlinear difference equations, vol. 4, Chapman and Hall / CRC, (2005).

[10] S. Kalabusic and M. R. S. Kulenovic, On the recursive sequence $x_{n+1}=\frac{\gamma x_{n-1}+\delta x_{n-2}}{C x_{n-1}+D x_{n-2}}$, J.difference. Equations
Appl., 9(8) (2003), 701-720.

[11] G. Karakostas and S. Stevic, On the recursive sequence $x_{n+1}=A+\frac{f\left(x_{n}, \ldots, x_{n-k+1}\right)}{x_{n-1}}, \quad$ Comm. Appl. Nonlinear Analysis, 11 (2004), 87-100.

[12] V. L. Kocic and G. Ladas, Global behavior of nonlinear difference equations of higher order with application, Kluwer Academic Publishers, Dordrecht, (1993).

[13] M. R. S. Kulenovic, G. Ladas and W. S. Sizer, On the recursive sequence $x_{n+1}=\frac{\alpha x_{n}+\beta x_{n-1}}{\chi_{n}+\delta x_{n-1}}$, Math. Sci. Res. Hot-Line 2(5) (1998), 1-16.

[14] M.R.S. Kulenovic, G. Ladas, Dynamics of second order rational difference equations with open problems and conjectures, Chapman \& Hall/CRC, Florida, (2001).

[15] M. Saleh and S. Abu-Baha, Dynamics of a higher order rational difference equation, Appl. Math. Comput; 181 (2006), 84-102.

[16] E. M. E. Zayed and M. A. EI-Moneam, On the rational recursive sequence $x_{n+1}=\frac{\left(\alpha x_{n}+\beta x_{n-1}+\gamma x_{n-2}+\delta x_{n-3}\right)}{\left(A x_{n}+B x_{n-1}+C x_{n-2}+D x_{n-3}\right)}, \mathrm{J}$. Appl. Math. nd Computing, 22 (2006), 247-262.

[17] E. M. E. Zayed and M. A. EI-Moneam, On the rational recursive sequence $\quad x_{n+1}=A x_{n}+\frac{\left(\beta x_{n}+2 x_{n-k}\right)}{\left(C x_{n}+D x_{n-k}\right)}$, Comm. Appl. Nonlinear Analysis, 16 (2009), No.3, 91 106. 